\title{
APRENDIZAJE DE LA LECTOESCRITURA EN ÉPOCA DE PANDEMIA
}

\section{LEARNING TO READ AND WRITE IN TIMES OF PANDEMICS}

\author{
Asdrúbal Emilfo Ayala Mendoza ${ }^{1}$ \\ Karolina Alexandra Gaibor Rios ${ }^{2}$
}

Recibido: 2021-05-25 / Revisado: 2021-06-20 / Aceptado: 2021-08-12 / Publicado: 2021-09-15

Forma sugerida de citar: Ayala-Mendoza, A. E. y Gaibor-Ríos, K. A. (2021). Aprendizaje de la Lectoescritura en Época de Pandemia. Retos de la Ciencia. 5(e). 13-22. https://doi.org/10.53877/rc.5.e.20210915.02

\section{RESUMEN}

El presente artículo de revisión titulado "El Aprendizaje de la Lectoescritura en Época de Pandemia", y en los que se determinan los factores que influyen y se suscitan a nivel educativo debido al confinamiento que ha generado dificultades en el aprestamiento de la lectoescritura. El objetivo que se plantea es sintetizar información bibliográfica sobre las distintas técnicas de lectoescritura que se desarrollaron en época de pandemia. Desde esta perspectiva, el estudio se orienta a una investigación bibliográfica documental con un análisis cualitativo, en la que se recabó información de distintas fuentes bibliográficas. Se analizan aportes de diversos autores, con varias perspectivas tanto en técnicas e importancia para determinar no solo los factores que inciden en dicho proceso, también las estrategias idóneas para el fortalecimiento de la lectoescritura que involucran ámbitos: institucional, familiar y estudiantil. Se concluye que la lectoescritura en tiempo de pandemia sobrellevo una serie de retos debido a que la educación se trasladó hacia la virtualidad con todas las exigencias y nuevas limitaciones que impone el distanciamiento físico para el desarrollo de un proceso de aprendizaje que de por sí requiere acompañamiento; la familia se convierte en el núcleo de la educación, ella aprendió a utilizar métodos lectores, estimuló el desarrollo de habilidades sociales, cognitivas, y afectivas necesarias para el desarrollo eficaz de la lectoescritura.

Palabras clave: aprendizaje, lectoescritura, niños, pandemia, familia.

\footnotetext{
${ }^{1}$ Magister en Psicopedagogía Talentos y Creatividad. Docente Investigador de la Universidad Tecnológica Indoamérica. Ecuador. E-mail: aayala2@indoamerica.edu.ec / ORCID: https://orcid.org/0000-0003-2444-176X

2 Tecnologo en Desarrollo del Talento Infantil. Estudiante Universidad Tecnológica Indoamérica. Ecuador. E-mail: karolinagaibor06@hotmail.com / ORCID: https://orcid.org/0000-0002-1002-5602
} 


\section{ABSTRACT}

This review article entitled "Literacy Learning in Pandemic Times", determines the factors that influence and arise at the educational level due to the confinement that has generated difficulties in the learning of literacy. The objective is to synthesize bibliographic information on the different literacy techniques that were developed during the pandemic period. From this perspective, the study is oriented to a documentary bibliographic research with a qualitative analysis, in which information was collected from different bibliographic sources. Contributions from various authors are analyzed, with several perspectives on techniques and importance to determine not only the factors that affect this process, but also the ideal strategies for strengthening literacy that involve institutional, family and student environments. It is concluded that reading and writing in times of pandemic brought with it a series of challenges due to the fact that education moved towards virtuality with all the demands and new limitations imposed by physical distance for the development of a learning process that in itself requires accompaniment; the family becomes the nucleus of education, it learned to use reading methods, stimulated the development of social, cognitive and affective skills necessary for the effective development of reading and writing.

Keywords: Learning, literacy, children, pandemic, family.

\section{INTRODUCCIÓN}

En los últimos tiempos las sociedades han experimentado grandes transformaciones en todos los ámbitos a una velocidad vertiginosa. Se suscitan múltiples cambios a nivel educativo, esto hecho fue evidente en el contexto actual por la emergencia sanitaria causada por el COVID-19.Se ha visto afectado el sistema educativo con un mayor impacto en la educación básica elemental ya que incidió en procesos asociados al aprendizaje especialmente la lectoescritura, por ello es necesario analizar el pensamiento de diversos autores sobre las implicaciones del tema a partir de la siguiente interrogante ¿Cómo ocurre el desarrollo de aprendizaje de la lectoescritura en época de pandemia?

En este período de pandemia la educación recurre a la implementación de estrategias como clases virtuales de forma sincrónica y asincrónica, en las que el estudio se desarrolla a distancia permitiendo la accesibilidad a la educación. El docente planifica actividades para que el niño y la familia construyan el conocimiento, sin duda las aplicaciones digitales se convirtieron en un eje fundamental en todo el proceso educativo. Entre las aplicaciones más utilizadas tenemos: Google presenta la aplicación Google Meet; Microsoft la aplicación Teams; y Eric Yuan (Zoom Video Communications) la plataforma virtual Zoom, las mismas fueron de gran utilidad, sin embargo esta migración hacia la virtualidad ha repercutido en el incremento de la dificultad para ofrecer los servicios educativos debido a que los estudiantes no cuentan con servicio de internet, esta circunstancia resulta un obstáculo en la educación pese a que las instituciones buscaron estrategias para continuar el aprendizaje conforme lo establece el sistema educativo (Salinas, D., 2020)

En tal sentido los efectos educativos que ocasiona la pandemia del coronavirus aún no se pueden medir con exactitud, pues todo es incierto. En tal sentido se menciona que todo parecía ser pasajero y que el confinamiento seria por un determinado tiempo, pero sin duda alguna esto trajo consigo varios cambios que 
afectaron a nivel educativo, económico, y emocional puesto que familias perdieron a sus padres, madres e hijos que en muchos casos eran el sustento económico de los hogares, y por la misma razón varios estudiantes no solo decayeron en su educación también otros abandonaron sus estudios. (Dussel, 2020)

De esta manera se incrementa el deterioro de la educación ya que los procesos educativos deberán replantearse constantemente para ajustarse a las circunstancias cambiantes que impone la pandemia, por ello es necesario trabajar en clases lúdicas creativas que cautiven el interés de los alumnos, las mismas deben estar ajustadas a la realidad de cada niño, a nuevas formas de evaluación y nuevas estrategias para trabajar con los padres de familia o representantes y la promoción del estudiante (Salinas, 2020).

Uno de los mayores conflictos que se ha detectado en el entorno educativo a raíz de la emergencia sanitaria ha sido los problemas en el aprendizaje de la lectoescritura, debido a que la educación se ha trasladado completamente al campo de la virtualidad, es decir el aislamiento ha obligado a implementar modalidades que se tenían como herramientas complementarias en la educación, esto implico que docentes y padres de familia tenga un rol activo en esta tarea que se ha tornado más ardua en este contexto. El padre de familia no siempre cuenta con el conocimiento necesario para brindar el apoyo requerido en la vida estudiantil de sus hijos y especialmente en el proceso de lectoescritura (Ceballos y Sevilla, 2020)

La lectoescritura es fundamental en la formación académica pues a través de ella la persona tiene autonomía para comprender el mundo, emitir criterios, transmitir ideas, que son la base para el desarrollo de procesos conductuales, sociales y cognitivos, y que raíz del confinamiento han ido disminuyendo su efectividad en el estudiante. Debido a la dificultad de estudiar virtualmente, la educación fue poco creativa e innovadora puesto que los usuarios no contaban con el conocimiento para la utilización de apps digitales y de conectividad, por lo que el aprendizaje no fue espontaneo y las actividades escolares no tuvieron la aplicación de los temas tratados especialmente en la lectoescritura. Actualmente ambos procesos se pueden potenciar con la ayuda de los cursos TIC que permiten a los docentes y estudiantes tener un acercamiento más dinámico en el proceso académico.

La lectoescritura se debe desarrollar con estrategias que sean agradables y dinámicas de tal manera que capte el interés de los niños y les motive a seguir participando activamente en el mencionado proceso. Esta época se distingue por el uso generalizado de la tecnología en función de múltiples proyectos, lo cual implica mayor facilidad de acceder a ella como vía principal para desarrollar las actividades de lectoescritura, sin embargo, el desafío es conseguirlo tomando en cuenta los requerimientos que la hagan más interesantes para los estudiantes.

Los procesos de lectoescritura no deben seguir en el ámbito tradicionalista que comúnmente se lo utilizaba ya que actualmente los estudiantes tienen nuevas formas de aprendizaje entre ellas la grafofonética la cual es una técnica que utiliza pictogramas para que el niño establezca relaciones y su vez vaya brindando respuesta a lo que va aprendiendo es decir va interiorizando la información. (Valenciano, 2019) Se considera un aspecto tan esencial para el proceso de aprendizaje la construcción de significados, el cual debe estar estrechamente relacionados con la interacción que se tiene el entorno, ello provoca un aprendizaje basado en la experiencia.

Debido a la pandemia, en Ecuador, se evidenció las grandes brechas que existen y afectan fundamentalmente el ámbito educativo, pues únicamente el $37 \%$ de cada 
hogar poseen acceso a internet, por lo que 4 de cada 10 familias poseen este recurso, (UNESCO, 2020). Las zonas rurales debido a la ubicación geográfica tienen mayor dificultad, en la mayoría de los casos deciden abandonar los estudios y dedicarse a las actividades del campo.

Por otro lado, 9 de cada 10 hogares cuentan con una línea de celular y/o televisor, a través de estos medios se puede acreditar un proceso de educación, pero a la vez no garantiza que toda la población estudiantil cuente con: datos móviles, televisión por cable, televisión en línea, servicio eléctrico y otros (UNESCO, 2020). Las empresas que proveían servicios de internet y televisión por cable tuvieron mayor demanda durante la crisis sanitaria el problema fue que los costos por estos servicios fueron altos y la perdida de fuentes de trabajo complico a las familias mantener el servicio o adquirirlo.

En la realidad ecuatoriana se agudizado los problemas relacionados al aprendizaje de la lectura y escritura debido a la migración al modelo virtual, por lo que se requiere prestar atención a esta problemática y conocer las causas que intervienen en el proceso de lectoescritura y a su vez buscar estrategias para erradicarlos.

Para Salinas (2020), existen diversas estrategias para fortalecer la lectoescritura entre los que menciona: a) La lectura mediada es una aplicación que trabaja emociones, pensamientos; y b) Libro álbum permite al niño interactuar en la historia brindando su propio punto de vista a partir de la imagen presentada. El objetivo de la presente investigación es determinar los factores que influyen en el aprendizaje de la lectoescritura en época de pandemia.

\section{METODOLOGÍA}

Para conocer las principales dificultades de lectoescritura que se presentaron durante la pandemia se realiza un análisis documental que cuenta con una profundidad descriptiva, con un enfoque cualitativo. El criterio de inclusión determinado analizó cada una de las referencias de la unidad de análisis, identificando los documentos que se encuentren de acuerdo con el tema. En el desarrollo de la investigación se utilizó un cuadro bibliográfico con el siguiente esquema: tipo de estudio, tipo de documento, título, autor, editorial, link, métodos, recursos e idea principal.

\section{RESULTADOS}

Se analizan los aportes de diversos autores entorno a la lectoescritura desde varias perspectivas para determinar no solo los factores que inciden en dicho proceso también las estrategias idóneas para su fortalecimiento.

En estudios realizados el docente tiene un rol importante, pues en el proceso de la lectoescritura se logró definir que cada vez los niños no enfocan su interés en la lectura, pues no tienen el acompañamiento pedagógico para el desarrollo de mencionado proceso y por ello presentan menos capacidades de concentración y comprensión de los textos que deben desarrollar en casa para el desarrollo de destrezas lingüísticas, lo que se relaciona con la ausencia del docente.

Salinas (2020), plantea la lectoescritura constituye procesos que necesitan ser practicadas de forma sistemática para así obtener la asimilación de los contenidos y de las temáticas. Para poder definir el gusto por leer se lo hace de manera autónoma, de tal forma que el aprendizaje no sea una obligación más bien sea un hábito adquirido. 
Valencia (2019), menciona dos escenarios que facilitan a los niños a desarrollar la lectoescritura: a) El entorno que rodea al niño ayuda a desarrollar un el aprendizaje mientras juega o se relaciona con los mismos va creando interés y al mismo tiempo va observando similitudes y diferencias y $b$ ) La relevancia que se genera desde lo vivido como experiencia hasta que se logra en cada niño establecer un proceso sistematizado de asimilación de la lectoescritura.

Otro punto de interés que resalta Valencia (2019), es que hay un conjunto de condiciones que preparan al alumno para el aprendizaje de la lectoescritura entre ellos tenemos: tener un lenguaje correcto, poseer una buena visión, discriminación de sonidos, percepción, coordinación, y una adecuada estabilidad emocional, todos los puntos mencionados son importantes para lograr un proceso de lectoescritura eficaz.

Estas se reconocen como expresas características de un verdadero proceso de lectoescritura en la que los niños en su plena etapa de aprendizaje logran desarrollar mayor o menor atención en dichos procesos de aprendizaje. Valencia alude otras condiciones a cumplir por parte del docente las mismas que contribuyen a que el desarrollo de lectura y escritura sea exitoso (Valencia, 2019), estas son: Impulsar de todas las maneras posibles que los alumnos logren un desarrollo completo, promover que las acciones de aprendizaje, y e interacción de dichos alumnos entorno a la comunicación, tomar en consideración el ritmo de los progresos de cada uno, gestionar motivación creando ambientes lúdicos, propiciar el desarrollo de la enseñanza aprendizaje.

Manejo del proceso de lectoescritura en tiempos de pandemia a través de la modalidad virtual.

En la presente era lo digital es un fuerte motor impulsor de nuevas experiencias de aprendizaje, sobre todo a través de las redes sociales como: WhatsApp, Facebook, YouTube y otros, para ello el uso de juegos interactivos alcanzan un peso mayor por su aporte a la nueva modalidad de estudios a distancia, es decir, desde casa. (Cassany, 2020)

Entre las estrategias para reforzar el proceso de la lectoescritura podemos mencionar los siguientes recursos digitales:

- Tato y sus palabras: es un recurso que estimula la parte cognitiva, está relacionada con la decodificación lectora en ella se presenta el avance de dicho desarrollo lectoescritor en tres diferentes niveles que se establecen en la dificultad del proceso y que son los que dan al trabajo en equipo o de manera individual, y la implementación de nuevas herramientas.

- Vamos a leer. - esta herramienta permite diferenciar letras y asociar palabras de forma lúdica y creativa.

- Libro álbum es una aplicación que permite tener un acercamiento real a la lectura, la misma está compuesta por imágenes la cual crea un dialogo entre el texto e imagen es decir las dos están fusionadas entre sí. (Salinas, 2020)

Las aplicaciones mencionadas permiten desarrollar puntos fundamentales como, por ejemplo: percepción visual, auditiva, asociación de palabras, vocabulario y atención. Tambien tenemos ciertos condicionamientos que se establecen en dichas aplicaciónes con las que se pretende alcanzar una opción propositiva a la lectoescritura y misma que induce a los estudiantes a tener interes, como ejemplo tenemos.

La lectura mediana, es una herramienta de lectura en la que el adulto tiene un rol importante pues se crea un vinculo donde trabaja emociones y su vez permite ser mas empatico e inclusivo. (Salinas, 2020) 
A continuación, se determina las investigaciones de distintos autores sobre procesos, métodos, estrategias para el desarrollo de la lectoescritura.

Cuadro 1:

Resumen de los principales aportes de las fuentes bibliográficas

\begin{tabular}{|c|c|}
\hline Autores & Título del artículo \\
\hline $\begin{array}{l}\text { Belda y García } \\
(2020)\end{array}$ & $\begin{array}{l}\text { "Lectoescritura en tiempos de } \\
\text { Covid. Libros interactivos de } \\
\text { las letras: propuesta de } \\
\text { innovación en el Grado de } \\
\text { Educación Infantil" }\end{array}$ \\
\hline
\end{tabular}

$\begin{array}{ll}\text { Bengtsson, A. et } & \text { "La casa convertida en } \\ \text { al (2020) } & \begin{array}{l}\text { mundo" como contexto de } \\ \text { aprendizaje en tiempos de } \\ \text { pandemia" }\end{array}\end{array}$

Cassany (2020) Enseñar en época de la COVID-19
Cevallos y Sevilla "Edel aislamiento social por el (2020)

Ceballos (2020)

covid-19 en la conciencia emocional y en la comprensión lectora. Estudio sobre la incidencia en alumnos con trastornos de aprendizaje y menor acceso a las nuevas tecnologías"

S. Sevilla y N. "La regulación emocional en niños para lograr la marravillosa aventura de la lectocomprensión en tiempos de pandemia"

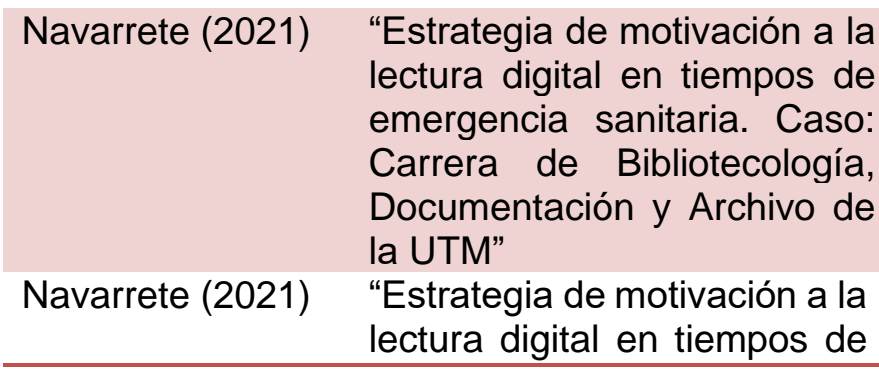

Este trabajo se enfoca en analizar las dificultades de enseñar en tiempos de pandemia y repensar en estrategias claves las cuales se puedan trabajar fonemas, grafemas que están principalmente enfocadas en los primeros años de educación básica pues es allí en donde el aprendizaje de la lectoescritura se volvió un gran reto.

Este estudio tiene el propósito reflexionar y acercarse al conocimiento de nuevas herramientas las cuales sean colaborativas es decir involucre a padres de familia $y$ estudiantes.

Como propósito realizar un análisis de como la cuarentena ha cambiado la manera de enseñar y aprender, así como las numerosas interrogantes que afectarán la educación en el futuro, con la aportación de la red, e incluso las limitaciones.

Este trabajo analiza la relación de la autorregulación emocional, conciencia emocional y lingüístico en la lectoescritura, de la misma manera conocer las dificultades que se presentaron en la pandemia.

Aquí se menciona el desarrollo lector enfocándose en que el niño no solo realice una simple lectura más bien debe leer comprender y entender para que puede tener un análisis que ayude a despejar dudas, sentimientos que pueda encontrar en la lectura.

Se enfoca en resaltar que lectura digital ha ayudado a potenciar los 
emergencia sanitaria. Caso: Carrera de Bibliotecología, Documentación y Archivo de la UTM"

$\begin{array}{lrl}\text { Navarro, } & \text { D. } & \text { "Enseñar a leer y escribir en } \\ \text { Lerner, } & \text { A. } & \text { pandemia" } \\ \text { Meneses, } & \text { K. } & \\ \text { López-Gil, } & \text { R. } & \\ \text { Artal y P. Otero } & \\ \text { (2020) } & \end{array}$

N. Valero, A. "Retos de la educación virtual Castillo, R. en el proceso enseñanza Rodríguez, M. aprendizaje durante la Padilla y M. pandemia de Covid" Cabrera, « -19 (2020)

D. Valencia(2019) los prerrequisitos para la
adquisión de
Lectoescritura" "Conocimiento Docente ante

conocimientos de la población como objeto de estudio y ha generado que se afronte de mejor manera la emergencia sanitaria que se vive actualmente.

Este estudio aborda todas las interrogantes que sacó a la luz la pandemia pues impuso cambios donde se difuminaron las aulas y el hogar se convirtió en la nueva escuela en la misma se presentan dificultades por desconocimiento de apps, tiempo, carencia servicio de internet lo cual deterioro el aprendizaje lectoescritor.

La presente investigación tiene como objetivo conocer los cambios que se realizaron a nivel educativo en el confinamiento causado por el COVID-19 en la misma se pretende implementar esquemas de lectoescritura para determinar falencias de lectura y escritura tomando en consideración la nueva de modalidad de estudios virtual que se está trabajando.

La intención de este documento es conocer el grado de capacitación y su vez las dificultades que los docentes presentaron para la enseñanza de lectoescritura en la pandemia, considerando que en el área administrativa de cada institución no estaba preparada para tal causa.

Nota: La tabla 1 determina las investigaciones de distintos autores sobre procesos, métodos, estrategias para el desarrollo de la lectoescritura.

\section{DISCUSIÓN}

Partiendo del análisis de los autores estudiados se pueden apreciar ciertos elementos de valor respecto al aprendizaje de la lectoescritura en tiempos de pandemia como el trabajo conjunto entre profesores, padres de familia y estudiantes, el funcionamiento coordinado de esa triada garantiza resultados eficientes en un proceso que por naturaleza requiere de acompañamiento.

Durante la emergencia sanitaria se implementó varios recursos entre ellos el currículo priorizado para las instituciones educativas, el mismo está enfocado en trabajar competencias y estrategias primordiales, pues si bien es cierto el aprendizaje se volvió un desafío no solo para los padres de familia también para las instituciones y alumnos para los cuales fueron afectados tanto económicamente y emocionalmente y es por ello que la educación necesita la solidaridad y empatía por parte de todos para lograr el éxito durante este cambio radical que desorientó a todos exigiendo adaptabilidad a esta nueva modalidad de estudios. (CEPAL-UNESCO, 2020) 
Los centros educativos deben desarrollar estrategias eficientes para la mejora de la lectoescritura utilizando recursos digitales que integren al padre de familia, docente y al niño. La casa es la nueva escuela y a través de la tecnología se convirtió en un lugar adecuado para desarrollar la nueva educación digital que ayuda adquirir habilidades lingüísticas propicias.

Se requiere flexibilización de la enseñanza debido a que se ha producido un cambio de modalidad hacia la virtualidad teniendo una transformación a una velocidad presurosa y sin tiempo para asimilar, por tal motivo van a la par procesos de reajustes y readaptación conjuntamente con la asimilación, y por otro no todos cuentan con el acceso a la tecnología hay desigualdad entre los estudiantes y esto constituye una realidad que tendrá impactos en los ritmos y el nivel de aprendizaje en los estudiantes.

Asimismo hay autores que promueven la iniciativa de que exista varios cambios en la comunidad educativa como la unificación y progreso de las habilidades en los medios digitales, tomando en cuenta la relevancia en el ámbito educativo sobre puntos importantes para la relación entre docentes y estudiantes, que les permite tener mejores virtudes, cualidades y destrezas a la hora de reencontrarse por este medio, en el cual consiente tener empatía, colaboración, respeto, y entre otros valores, y aún más lograr adaptarse a los nuevos cambios en la educación, sin perder lo esencial de la educación.

Esta pandemia ha traído grandes cambios en el ámbito de la lectoescritura, y sobre todo para los docentes porque el desarrolla los temas de enseñanza para los primeros años de escuela. Los docentes aplican metodología concreta para lograr un mejor desarrollo preestablecido en la temática impartida hacia los estudiantes, logrando resultados de un aprendizaje de acuerdo con su ritmo de aprendizaje en el proceso educativo.

Es por ello importante emplear estrategias y métodos que despierten una motivación intrínseca en los estudiantes, que a su vez impliquen una dinámica interactiva y participativa, donde el estudiante sea activo en la construcción del aprendizaje y que refleje cercanía pese al distanciamiento físico que impone la pandemia. En tal sentido se concluye que:

En el estudio del aprendizaje de la lectoescritura en época de pandemia se han aplicado estrategias en las que el docente ayudado por los padres de familia trabajó conjuntamente en el manejo de Apps y libros, lúdicos que son más amigables para el niño en el aprestamiento de la lectoescritura.

Los componentes que inciden en la lectoescritura en tiempos de pandemia fueron determinados de acuerdo a la revisión de numerosos estudios, los cuales coinciden en señalar como ejes centrales la importancia del profesor en este proceso, así como de los padres y la coordinación de ambos actores para el éxito en el aprendizaje de la lectoescritura en los alumnos, otro aspecto fundamental es la necesidad de que se cumplan ciertos prerrequisitos que garantizan la adquisición de la lectoescritura, y que los métodos, estrategias utilizadas durante el aprendizaje de la lectoescritura sean interactivos, motivadores y que brinden un acercamiento a la realidad.

La educación actual tiene como principal desafío propiciar canales alternativos de acceso a internet para quienes carecen de estos servicios puedan acceder a la enseñanza en línea y recibir las indicaciones que permitan a sus familiares guiarles correctamente en este proceso tan importante. 


\section{REFERENCIAS BIBLIOGRÁFICAS}

Belda, M., \& García, G. (2020). Lectoescritura en tiempos de covid. Libros interactivos de las letras: propuesta de innovación en el Grado de Educación Infantil. EDUNOVATIC, $1(1), \quad 1-4$. https://repositorioinstitucional.ceu.es/bitstream/10637/12826/1/Lectoescritura Belda_EDUNOVATIC_2020.pdf.

Bengtsson, A., Bugallo, L., Coccoz, V., D’Adamo, P., Lozada, M., Méndez, L., . . . Santini, I. (2020). "La casa convertida en mundo" como contexto de aprendizaje en tiempos de pandemia. Ruta Maestra Edición 29, 1(1), 36-46. https://ri.conicet.gov.ar/bitstream/handle/11336/115938/CONICET_Digital_Nr o.83076711-eea8-4860-9c48-cfba292f5802 A. pdf? sequence=2\&isAllowed=y.

Cassany, D. (2020). Enseñar en época de la COVID-19. El educador. Un punto de encuentro., $1(1)$, 4-11. Obtenido de https://www.normainfantilyjuvenil.com/co/uploads/2020/07/revista-eleducador-julio2020.pdf

Castillo, C. (2018). Análisis comparativo del proceso de aprendizaje de la lectoescritura en dos contextos educativos diferentes. Morelos, México: Universidad Autónoma del Estado de Morelos. Tesis de Maestría en Atención a la Diversidad y Educación Inclusiva.

Ceballos, N. ; Sevilla, S. (2020). El efecto del aislamiento social por el covid-19 en la conciencia emocional y en la comprensión lectora. Estudio sobre la incidencia en alumnos con trastornos de aprendizaje y menor acceso a las nuevas tecnologías. Revista internacional de Educación para la Justicia Social, 9(3), 113.

https://ebuah.uah.es/xmlui/bitstream/handle/10017/42866/efecto_sevilla_RIEJ S_2020.pdf? sequence $=3$ \&isAllowed $=y$.

CEPAL-UNESCO. (2020). La educación en tiempo sde la pandemia de

Dussel, I. (2020). La formación docente y los desafíos de la pandemia. Revista Académica Digital (EFI), 6(10), 11-25. Obtenido de https://ppct.caicyt.gov.ar/index.php/efi/article/view/18585/45454575770497>

Meneses, A., Gallegos, F., Claro, M., Muller, M., Murillo, F., \& Sotomayor, C. (2020). Didácticas para la proximidad: Aprendiendo en tiempos de crisis. Santiago de Chile.

https://www.pucv.cl/uuaa/site/docs/20200723/20200723181922/dida_cticas_ para_la_proximidad_aprendiendo_en_tiempos_de_crisis.pdf: Propuestas Educación Mesa Social COVID-19.

https://revistas.utm.edu.ec/index.php/Rehuso/article/view/2988/3109.

Navarro, F., Lerner, D., Meneses, A., López-Gil, K., Artal, R., \& Otero, P. (2021). Enseñar a leer y escribir en pandemia. Textos. Didáctica de la Lengua y la Literatura., 92(1),

57-62. https://d1wqtxts1xzle7.cloudfront.net/66289680/Navarro_et_al_2021_Ensenar a_leer_y_escribir_en_pandemia_PREPRINT.pdf?1618693844=\&responsecontent-

disposition=inline\%3B+filename\%3DEnsenar_a_leer_y_escribir_en_pandemi a.pdf\&Expires $=1626877097 \&$ Signatur.

Salinas, D. (2020). Fortalecimiento de los procesos de lectura y escritura en tiempos de pandemia. Obtenido de https://repository.javeriana.edu.co/bitstream/handle/10554/54378/TESIS\%20 
DANA\%20DURLEY\%20SALINAS\%20CRUZ\%20PUJ.pdf?sequence=1\&isAllo wed $=y$

Salinas, D. (2020). Fortalecimiento de los procesos de lectura y escritura en tiempos de pandemia. Colombia: Universidad https://repository.javeriana.edu.co/bitstream/handle/10554/54378/TESIS\%20 DANA\%20DURLEY\%20SALINAS\%20CRUZ\%20PUJ.pdf?sequence=1\&isAllo wed $=\mathrm{y}$.

Salinas, D. (2020). Fortalecimiento de los procesos de lectura y escritura en tiempos de pandemia. Colombia: Universidad https://repository.javeriana.edu.co/bitstream/handle/10554/54378/TESIS\%20 DANA\%20DURLEY\%20SALINAS\%20CRUZ\%20PUJ.pdf?sequence=1\&isAllo wed $=\mathrm{y}$.

UNESCO. (2020). La educación en tiempos de la pandemia COVID-19. USA.: UNESCO Biblioteca digital. Obtenido de https://unesdoc.unesco.org/ark:/48223/pf0000374075?posInSet=1yqueryld=6 606d041-e555-4f06-b4c4-42ea1b4153e9

Valencia, D. (2019). Conocimiento Docente ante los prerrequisitos para la adquisión de la Lectoescritura. Panama: Universidad Especializada de las Américas. Tesis de Maestría en Psicopedagogía con especialización en Dificultades en el Aprendizaje.

Valenciano. (2019). La metáfora como alternativa metodológica para investigar y reflexionar acerca de la práctica pedagógica en la enseñanza de la lectoescritura. Educare, 5.

Valenciano, G. (2019). La metáfora como alternativa metodológica para investigar y reflexionar acerca de la práctica pedagógica en la enseñanza de la lectoescritura. Revista Electrónica Educare, 23(1), 1-23. . Obtenido de https://dialnet.unirioja.es/servlet/articulo?codigo=7002097

Valenciano-Canet, G. (2019). La metáfora como alternativa metodológica para investigar y. Scielo, 23(1), 2.

Valero, Nereida. (2020). Retos de la educación virtual en el proceso enseñanza aprendizaje durante la pandemia de Covid-19. Dominio de las Ciencias, 6(4), 1201-1220.

https://dominiodelasciencias.com/ojs/index.php/es/article/view/1530.

Velázquez, M. A. (2016). Lectoescritura: eventos de literacidad enpreescolar. Revista Iberoamericana, 6(12), 6. Recuperado el Jueves de 2021 de 2021, de http://www.ride.org.mx/index.php/RIDE/article/view/208/922

Zamorano, C. A. (Mayo de 2019). Recursos eduactivos digitales. (Gobierno de Canarias) Recuperado el Lunes de 2021 de 2021, de https://www3.gobiernodecanarias.org/medusa/ecoescuela/recursosdigitales/2 019/05/16/vamos-a-leer-2/ 